computed tomography scan, which it showed brain metastasis. He expired 9 months later after initial diagnosis. Altogether, six cases of EATL with intracranial metastasis were reviewed for their clinical characteristics.

Results The mean age was 54 years old (range from 35 to 65 years old). Two-thirds were males. Many (two-thirds) were without a history of celiac disease. One-half of the patients had abdominal pain as the initial gastrointestinal presentation. The primary lesion site was in the small intestine with jejunum predominance (66.6\%). Eighty-three percent of patients had abdominal surgery and adjuvant chemotherapy for the primary lesion. The majority site of intracranial metastasis was supratentorial V.S. infratentorial; 83\% V.S 17\%. Half of intracranial metastasis $(50 \%)$ presented with a change of mental status. Headache (33.3\%) and weakness of extremities $(33.3 \%)$ were the next most common presentations of metastasis. Radiotherapy, chemotherapy and steroid therapy were used to treat intracranial metastasis. The survival was an average of 9.6 months (3 months to 16 months) after the initial diagnosis of EATL.

Conclusions The prognosis of EATL with intracranial metastasis is generally poor with a mean survival time of fewer than 10 months.

\section{IDDF2018-ABS-0170 SINGLE CENTRE INITALI EXPERIENCE OF EUS-GUIDED GALLBLADDER DRAINAGE FOR CHOLECYSTITIS USING A LUMEN APPOSING METAL STENTS (LAMS), FOR NONSURGICAL CANDIDATES}

Adeel Urrehman*, Christopher JL Khor, Yung Ka Chin, Ravishankar Asokkumar. Singapore General Hospital, Singapore

\subsection{6/gutjnl-2018-IDDFabstracts.137}

Background With the introduction of new LAMS, EUS guided gallbladder $(\mathrm{GB})$ drainage is now becoming a viable alternative to percutaneous GB drainage with favourable clinical success rates and potentially fewer adverse events.

Methods Patients with cholecystitis who were not surgical candidates due to advanced malignancy or severe co-morbidities underwent EUS guided GB drainage in a single centre from April 2017 till OCT 2017. LAMS $15 \mathrm{mmx10} \mathrm{mm} \mathrm{with} \mathrm{the} \mathrm{electrocautery-}$ enhanced delivery system were used in all patients.

Results A total of 6 patients with a median age was 65 years (range, 58-93). Three patients had severe cardiovascular disease and rest had advanced hepatobiliary malignancy. The Charlson Comorbidity Index median value 8 (4-10). Cholecystitis was graded moderate in three patients and mild in three per the Tokyo guidelines. Three patients were performed as primary GB drainage, and three were performed as endoscopic internalisation of prior percutaneous biliary drainage. LAMS deployment was technically successful in four patients. Two patients had contrast extravasation on the initial filling of GB via the percutaneous drain and after needle puncture respectively. As the GB was not distended in each case, the respective procedures were aborted and referred for surgical management. Of the four deployed stents, one had the proximal flange obstructing the pylorus, and this was treated with another LAMS deployed at the pylorus to prevent gastric outlet obstruction. There was no periprocedural complication. The median duration of the successful procedures was 33.5 min (20-83). Three patients had advanced malignancy, so the LAMS was left permanently. One patient had transmural stone extraction through the LAMS. During this procedure, a stone migrated into the common bile duct. This patient underwent ERCP and LAMS removal at one month after placement. One patient had severe abdominal pain due to postprocedure bile leak and was treated conservatively with intravenous antibiotics. The overall success rate was $67 \%(4 / 6)$. Of the four successfully deployed LAMS, clinical success was observed in all.

Conclusions GB drainage using LAMS is technically feasible for non-surgical candidates with cholecystitis. Inability to distend the GB adequately for safe LAMS deployment was the main reason for the failure of the procedure.

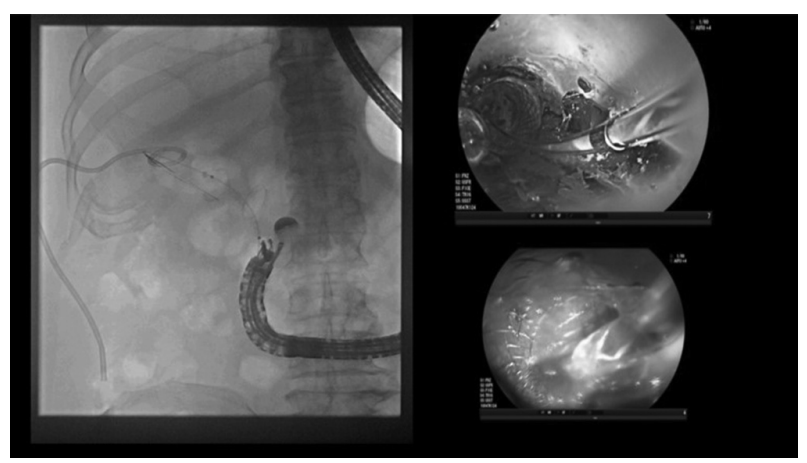

Abstract IDDF2018-ABS-0170 Figure 1

\section{IDDF2018-ABS-0171 EUS-GUIDED GASTROJEJUNOSTOMY USING A LUMEN APPOSING METAL STENT IN PATIENTS WITH SYMPTOMATIC GASTRIC OUTLET OBSTRUCTION}

Adeel Urrehman*, Christopher JL Khor, Yung Ka Chin, Ravishankar Asokkumar, Damien MY Tan. Singapore General Hospital, Singapore

\subsection{6/gutjnl-2018-IDDFabstracts. 138}

Background EUS gastrojejunostomy (GJ) is emerging as an alternative novel technique in patients with symptomatic gastric outlet obstructions (GOO) who have advanced malignancy. Recent studies have shown that the EUS-guided approach has less adverse events and is more cost-effective compared to laparoscopic GJ. We share our experience of 5 patients who underwent EUS Guided GJ with favourable outcomes.

Methods 5 patients with symptomatic gastric outlet obstruction underwent EUS-guided GJ interventions from May. 2017 to Nov. 2017. Patients were informed of the potential risks and informed consent was taken. Technical success was defined as successful deployment of lumen apposing metal stents (LAMS); while clinical success was the ability to tolerate diet.

Results Median age was 61.5 (Range 53-83) years. GOO was secondary to advanced pancreatic malignancy (4) and duodenal malignancy (1). Two patients had altered anatomy from previous post bilroth gastrectomy and Roux-en-Y hepaticojejunostomy. For all five patients with GOO, LAMS $15 \mathrm{~mm}$ diameter with electrocautery-enhanced delivery system was used to create the GJ anastomosis. Identification of the distal jejunal limb was done with an inflated balloon catheter and this was use as a target for direct puncture with a $19 \mathrm{G}$ needle. All five interventions had technical success with median procedure time of $80 \mathrm{~min}$ (38-163 range). All stents were dilated up to 